
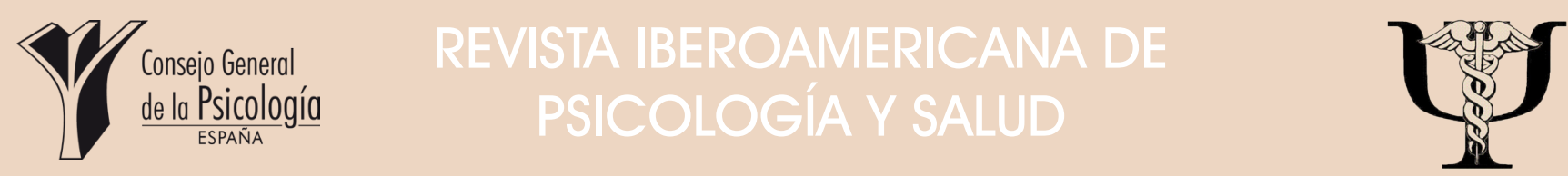

Revista Oficial de la Federación Iberoamericana de Asociaciones de Psicología (FIAP)

[Official Journal of the Latin-American Federation of Psychological Associations]

\title{
Ranking de investigación de las universidades públicas españolas.
}

\section{Gualberto Buela-Casal ${ }^{1 *}$, Alejandro Guillén-Riquelme', Tamara Ramiro-Sánchez y Raúl Quevedo-Blasco'}

'Centro de Investigación Mente, Cerebro y Comportamiento (CIMCYC). Universidad de Granada, Granada, España.

- Recibido: 17 - Septiembre - 2016 . Aceptado: 7 - Noviembre - 2016

\section{RESUMEN}

La evaluación de la actividad investigadora se hace necesaria año tras año para conocer qué aspectos se relacionan con una mejora en la producción y la productividad científica. El objetivo de este estudio es actualizar, con los datos del año 2014, el ranking de investigación de las universidades públicas españolas. Se siguió la misma metodología que en ediciones anteriores. Se evaluó en cada una de las universidades públicas españolas la producción y productividad en investigación a través de siete criterios (artículos en revistas indexadas en el JCR, tramos de investigación, proyectos I+D, tesis doctorales, becas FPU, doctorados con Mención hacia la Excelencia y patentes) y se obtuvo también un ranking global formado por estos indicadores (tanto en producción, como en productividad). En el ranking global, las universidades con una mayor producción son Barcelona, Complutense de Madrid y Granada. Los primeros puestos en productividad están ocupados por la Pompeu Fabra, Autónoma de Barcelona y Pablo de Olavide. Se puede observar que las primeras universidades en productividad apenas varían entre ediciones, lo que indica que los factores organizacionales y las políticas de dichas universidades dan resultados positivos.

PALABRAS CLAVE: Ranking, Producción Científica, Productividad Científica, Investigación, Universidades Públicas Españolas.

\section{ABSTRACT}

Research ranking of Spanish public universities. Year after year it is necessary to evaluate research activity in order to ascertain which aspects are related to an improvement in scientific production and productivity. The objective of this study is to update the research ranking of Spanish public universities, based on data from 2014. The same methodology employed in previous editions was followed. Research production and productivity were evaluated for each of the Spanish public universities based on seven criteria (articles in journals indexed in the JCR, research periods, R+D projects, doctoral theses, FPU (training of university professors) grants, doctoral studies awarded with a citation of excellence and patents) and a global ranking was also obtained based on these indicators (both in terms of production and productivity). In the global ranking, the universities with a higher production are Barcelona, Complutense of Madrid, and Granada. In terms of productivity, the first positions are held by the universities Pompeu Fabra, the Autonomous University of Barcelona and Pablo de Olavide. It can be observed that the top universities in terms of productivity have barely varied between editions, indicating that organizational factors and the policies of said universities have yielded positive results.

KEYWORDS: Ranking, Scientific Production, Scientific Productivity, Research, Spanish Public Universities.

En los últimos años, debido a los cambios producidos en el sistema universitario español tras la adaptación al Espacio Europeo de

*Correspondencia: Gualberto Buela-Casal

Centro de Investigación Mente, Cerebro y Comportamiento (CIMCYC).

Universidad de Granada. Campus de Cartuja, s/n.

Código Postal: 18011 , Granada (España).

E-mail:gbuela@ugr.es

(C) 2017 Sociedad Universitaria de Investigación en Psicología y Salud. Publicado por Consejo General de Colegios Oficiales de Psicólogos, España. Este es un artículo Open Access
bajo la CC BY-NC-ND licencia (http://creativecommons.org/licencias/by-nc-nd/4.0/)
Educación Superior (EEES) y los recursos e inversiones realizados, se ha hecho necesaria la elaboración de estudios e informes que evalúen la calidad de la educación superior y la investigación en las universidades en general, y en concreto de las españolas. Prueba de ello son los informes publicados periódicamente, provenientes de organismos como el Ministerio de Educación, Cultura y Deporte (2015a, 2015d), el Ministerio de Ciencia e Innovación 
(2015) o la Conferencia de Rectores de las Universidades Españolas (CRUE, 2015). También son numerosos los estudios que analizan la adaptación al EEES, tanto desde la perspectiva del profesorado (Ariza, Quevedo-Blasco y BuelaCasal, 2014; Quevedo-Blasco, Ariza y BuelaCasal, 2015), como del alumnado (RamiroSánchez, Bermúdez y Buela-Casal, 2016).

Este interés por la evaluación de la calidad de las universidades, se hace evidente con el análisis y desarrollo de sistemas de información y clasificaciones (Berbegal-Mirabent y RibeiroSoriano, 2015). El objetivo de esta constante evaluación es proporcionar información y transparencia académica e investigadora por parte de las universidades; un ejemplo específico se puede ver en las universidades públicas valencianas (Pérez, Pastor y Peraita, 2015). La actividad investigadora del sistema universitario español también ha sido objeto de evaluación, teniendo en cuenta diversos criterios (De Filippo, García-Zorita, Lascurain-Sánchez, Marugán y Sanz-Casado, 2014; de MoyaAnegón, 2014; González Sala y Osca Lluch, 2016; Velasco, Vilariño, Amado y Fariña, 2014). Asimismo, en estudios recientes se ha analizado la eficiencia en investigación en base a aspectos económicos (Buela-Casal, Bermúdez, Sierra, Guillén-Riquelme y Quevedo-Blasco, 2015). No sólo las instituciones sino también las revistas científicas españolas han sido analizadas en algunos estudios (García-Pereira y QuevedoBlasco, 2015) o la composición de los comités editoriales de las revistas científicas (GonzálezSala, Fonseca-Baeza y Osca-Lluch, 2014).

En los útimos años se ha producido una proliferación de estudios en los que se analizan las características de las medidas bibliométricas, estableciendo que los distintos indicadores bibliométricos no funcionan igual, según el campo de conocimiento de aplicación (Hirsch y Buela-Casal, 2014). De esta forma se ha producido un aumento en el número de tesis doctorales relacionadas directamente con la bibliometría (Osca-Lluch, Haba, Fonseca, Civera y Tortosa, 2013). No obstante, también se debe tener presente las críticas recibidas al actual procedimiento de publicación (Buela-Casal, 2014), a los sistemas integradores de producción científica (Olivas-Ávila y Musi-Lechuga, 2014) y a los propios criterios de calidad (FernándezRíos y Rodríguez-Díaz, 2014; García, 2014).
A partir de la elaboración de rankings de universidades se obtiene información sobre la actividad científica que permite orientar la toma de decisiones sobre posibles estrategias de mejora, fortalecimiento, o distribución de los recursos financieros en cuanto a los criterios evaluados. Diversos rankings se realizan periódicamente, lo que también permite observar la evolución en el tiempo de las diferentes universidades, tanto a nivel internacional (Journals Consortium, 2015; Leiden Ranking, 2015; QS World University Rankings, 2015; Ranking Web of Universities, 2015; SCIMAGO, 2015; Shangai Jiao Tong University, 2015; Times Higher Education, 2016; U-Multirank, 2015) como nacional (Buela-Casal, Quevedo-Blasco y Guillén-Riquelme, 2015; Pérez y Aldás, 2015; Torres-Salinas, Delgado López-Cózar, Robinson García, Triguero y Herrera, 2014).

El objetivo del presente artículo es actualizar el ranking de investigación de las universidades públicas españolas (Buela-Casal et al., 2015) con los datos relativos al año 2014 (o los más recientes disponibles). Así, se evaluó en cada una de las universidades la producción y la productividad en investigación, tanto de forma específica (en cada uno de los siete indicadores objeto de estudio), como de forma global (a través de estos indicadores ponderados).

\section{MÉTODO}

\section{- DISEÑO}

Se trata de un estudio descriptivo mediante análisis de documentos que ha sido elaborado y redactado siguiendo los Principios de Berlín para Rankings de Instituciones de Educación Superior (International Ranking Expert Group, 2006).

\section{- UNIDADES DE ANÁLISIS}

Se analizaron siete criterios en cada universidad pública: artículos publicados en revistas indexadas en el Journal Citation Reports (JCR), tramos de investigación, proyectos $I+D$, tesis doctorales, becas FPU, doctorados con Mención hacia la Excelencia y patentes (tanto registradas como explotadas). Se utilizaron las fuentes del año 2014 o, si no estaban disponibles, las más recientes para obtener la información de cada uno de los indicadores. Asimismo, se seleccionó 
el intervalo temporal necesario para analizar de forma adecuada cada criterio (ver Tabla 1).

Tabla 7

Criterios e indicadores utilizados en el estudio

\begin{tabular}{|c|c|}
\hline Criterio & Indicador \\
\hline $\begin{array}{l}\text { Artículos publicados } \\
\text { en revistas indexadas } \\
\text { en el Journal Citation } \\
\text { Reports (JCR) }\end{array}$ & $\begin{array}{l}\text {-Producción. Número de artículos publicados, en el } \\
\text { año } 2014 \text {, en revistas de la Colección principal de Web } \\
\text { of Science (WoSs indexadas en el JCR. } \\
\text {-Productividad. Proporción de artículos publicados, } \\
\text { en el año } 2014 \text {, en la Colección principal de WoS S } \\
\text { de revistas indexadas en el JCR por profesores } \\
\text { funcionarios. }\end{array}$ \\
\hline $\begin{array}{l}\text { Tramos de } \\
\text { investigación (TI) }\end{array}$ & $\begin{array}{l}\text {-Producción. Número total de sexenios de } \\
\text { investigación obtenidos por los profesores funcionarios } \\
\text { (curso 2014/2015). } \\
\text {-Productividad. Proporción de tramos de investigación } \\
\text { obtenidos por los profosores funcionarios dividido entre } \\
\text { el número total de profesores funcionarios. }\end{array}$ \\
\hline Proyectos I+D & $\begin{array}{l}\text {-Producción. Número de proyectos I+D concedidos a } \\
\text { cada universidad en la convocatoria de } 2017 \text { (tanto en } \\
\text { la modalidadd de Excelencia, como de Retos). } \\
\text {-Productividad. Proporción de proyectos I+D } \\
\text { (modalidadades de Excelencia y Retos) en la } \\
\text { convocatoria de } 20144 \text { dividida entre el número total } \\
\text { de profesores funcionarios. }\end{array}$ \\
\hline Tesis doctorales & $\begin{array}{l}\text {-Producción. Número de tesis doctorales defendidas } \\
\text { en un período de cinco cursos (desde el curso } \\
2009 / 2010 \text { hasta el curso } 2013 / 2014 \text { ). } \\
\text {-Productividad. Proporción de tesis doctorales } \\
\text { defendidas, por profesores funcionarios en un periodo } \\
\text { de cinco cursos (desde el curso } 2009 / 2010 \text { hasta el } \\
\text { curso 2013/2014). }\end{array}$ \\
\hline Becas FPU & $\begin{array}{l}\text {-Producción. Número de becas FPU concedidas en la } \\
\text { convocatoria de } 2014 \text {. } \\
\text {-Productividad. Proporción de becas FPU en la } \\
\text { convocatoria de } 2014 \text { dividido entre el número total } \\
\text { de profesores funcionarios. }\end{array}$ \\
\hline $\begin{array}{l}\text { Doctorados con } \\
\text { Mención hacia la } \\
\text { Excelencia }\end{array}$ & $\begin{array}{l}\text {-Producción. Número de doctorados con Mención } \\
\text { hacia la Excelencia (convocatoria de 2011) de cada } \\
\text { universidad. } \\
\text {-Productividad. Proporción de doctorados con } \\
\text { Mención hacia la Excelencia en la convocatoria de } \\
2011 \text { dividido entre el número total de profesores } \\
\text { funcionarios (de lo datos del ranking 2013). }\end{array}$ \\
\hline Patentes & $\begin{array}{l}\text {-Producción. Número de patentes registradas en el } \\
\text { periodo } 2010-2014 \text { y explotadas en el periodo } 2004 \text { - } \\
2008 . \\
\text {-Productividad. Proporción de patentes registradas } \\
\text { de } 2010 \text { a } 2014 \text { y explotadas de } 2004 \text { a } 2008 \text { por } \\
\text { profesores funcionarios. }\end{array}$ \\
\hline & $\begin{array}{l}\text { uyen a Catedráticos de Universidad } \\
\text { itaria [CEU] y Profesores Titulares de } \\
\text { urso 2014/2015. }\end{array}$ \\
\hline
\end{tabular}

\section{- PROCEDIMIENTO}

Para el cálculo de la producción y productividad se siguió el mismo procedimiento que el utilizado por Buela-Casal et al. (2015) en todos los criterios, excepto en los tramos de investigación. Para la producción se analizaron los datos actualizados del curso 2014/15; en el cálculo de la productividad, en el presente estudio, a diferencia de ediciones anteriores en las que la productividad de los tramos de investigación se calculaba a través del Índice $S$ (IS: número de tramos de investigación obtenidos por los profesores funcionarios dividido por el número total de tramos de investigación posibles de esos funcionarios), se utiliza la proporción de tramos de investigación (de los profesores funcionarios) por profesorado funcionario en el curso 2014/15. Esto se debe a que no se dispone de datos actualizados sobre el IS, información obtenida por el informe de la Comisión Nacional de Evaluación de la Actividad Investigadora (CNEAI, 2009), y utilizada en los rankings anteriores de investigación de las universidades públicas españolas de 2009 a 2013.

\section{- PESO DE LOS INDICADORES}

En este ranking, al igual que en los anteriores, se le otorgó un peso a cada uno de los siete indicadores analizados (véase Tabla 2). Los pesos de seis indicadores (artículos JCR, tramos de investigación, proyectos $I+D$, tesis, becas FPU y doctorados) se obtuvieron del ranking de productividad en investigación de las universidades públicas españolas de 2008 (Buela-Casal, Bermúdez, Sierra, Quevedo-Blasco y Castro, 2009). El peso del séptimo indicador (patentes) se obtuvo del estudio de Buela-Casal y Sierra (2007), en el que, a través de una encuesta aplicada a una muestra representativa de Catedráticos de Universidad y Profesores Titulares de Universidad (con al menos dos y un tramo de investigación respectivamente), se valoraba el peso de cada criterio para evaluar la producción en la investigación científica en España. Se utilizó la escala tipo Likert con un rango de 1 a 5, como en las ediciones anteriores (de 2009 a 2013).

Tabla 2

Pesos para cada uno de los criterios utilizados en el estudio

\begin{tabular}{|l|l|}
\hline Criterio & Valor \\
\hline $\begin{array}{ll}\text { Artículos publicados en revistas indexadas en el } \\
\text { JCR }\end{array}$ & 4,19 \\
Tramos de investigación & 3,95 \\
Proyectos I+D & 3,90 \\
Tesis doctorales & 3,47 \\
Becas FPU & 3,02 \\
Doctorados con Mención hacia la Excelencia & 3,02 \\
Patentes & 2,67 \\
\hline Nota. Puntuaciones en una escala tipo Likert de 1 a 5. & \\
\hline
\end{tabular}




\section{- PARA ESTABLECER LA PRODUCCIÓN}

Todas las búsquedas fueron realizadas entre los días 4 y 11 de febrero de 2016 . Se realizaron por dos investigadores de forma independiente para garantizar la fiabilidad, alcanzando para cada uno de los indicadores un acuerdo total. Las fuentes empleadas para cada indicador fueron:

\section{a) Artículos publicados en revistas indexadas} en el JCR: se realizó la búsqueda de los artículos publicados en cada universidad pública en el año 2014 en la "Colección principal de Web of Science" (WoS). Para ello, en primer lugar, se seleccionó el campo de búsqueda de "Organizaciones-Nombre preferido" dentro de la WoS (Colección principal), y posteriormente se seleccionó la herramienta "Seleccionar del índice" para buscar cada universidad empleando la abreviatura "Univ*" seguido del nombre de cada universidad. Una vez se añade el registro de la universidad pertinente, automáticamente, se realiza la búsqueda de todas las posibles variantes o combinaciones de cada universidad, registradas en la WoS (Colección principal). Finalmente, la búsqueda se refinó por tipo de documento, seleccionando únicamente los "artículos" y las "revisiones".

b) Tramos de investigación: se calculó a través del número de tramos de investigación totales obtenidos por los profesores funcionarios (CU, CEU y PTU) de cada universidad según información del curso 2014/15. Para ello, se utilizaron los datos de la "Estadística de personal de las universidades: Personal Docente e Investigador", del Ministerio de Educación, Cultura y Deporte (2015b).

c) Proyectos I+D: se contabilizó el número de proyectos concedidos por el Ministerio de Economía y Competitividad en la convocatoria de 2014 a cada universidad pública española. Se tuvieron en cuenta tanto los proyectos en la modalidad de Excelencia [Programa Estatal de Fomento de la Investigación Científica y Técnica de Excelencia], como de Retos [Programa Estatal de I+D+i Orientada a los Retos de la Sociedad].

d) Tesis doctorales: para la búsqueda se empleó la base de datos de tesis doctorales realizadas en las universidades españolas TESEO (del Ministerio de Educación, Cultura y Deporte). En el campo "Universidad" se seleccionó cada una de las universidades públicas españolas y en el campo "Curso académico" se señaló el intervalo desde el 2009-2010 hasta el 20132014. En el presente estudio, a diferencia de ediciones anteriores, se tuvo en cuenta hasta el curso 2013-14 dado que en esta edición del ranking el periodo temporal (entre el último curso analizado y el momento de la búsqueda) ha sido lo sufientemente amplio como para que estén incorporadas todas las tesis de dicho curso académico. A esto se añade que actualmente, en la mayoría de las universidades, las tesis doctorales defendidas son incorporadas casi de inmediato a TESEO.

e) Becas FPU: se contabilizó el número de becas concedidas a cada universidad en la convocatoria 2014 (a través de las dos resoluciones publicadas: del 20 de agosto de 2015 y del 30 de octubre de 2015) por el Ministerio de Educación, Cultura y Deporte.

\section{f) Doctorados con Mención hacia la} Excelencia: se contabilizó el número de doctorados con Mención hacia la Excelencia concedidos por el Ministerio de Educación (Secretaría General de Universidades) a cada universidad en la única convocatoria (correspondiente al 2011, a través de las dos resoluciones publicadas: del 6 de octubre de 2011 y del 7 de junio de 2012).

g) Patentes: para el cálculo de este indicador se tuvieron en cuenta tanto las patentes registradas como las explotadas de cada universidad pública española en un periodo de cinco años. Se atendió a la información disponible más actualizada: 2010-2014 para las patentes registradas (información obtenida de la Base de Datos de la Oficina Española de Patentes y Marcas, OEPM); y 2004-2008 para las patentes explotadas (datos obtenidos del informe "La universidad española en cifras" [CRUE, 2011]). $\mathrm{Al}$ igual que en los rankings anteriores, se le otorgó un punto a cada patente explotada (debido a la producción de resultados y, por tanto, a la obtención de beneficios) y de 0,5 a cada una de las registradas. Para obtener el valor total de cada universidad se sumaron estos dos valores. 


\section{- PARA ESTABLECER LA PRODUCTIVIDAD}

En primer lugar, se utilizó la fuente actualizada del curso 2014/15 sobre "Estadística de personal de las universidades: Personal Docente e Investigador del Ministerio de Educación, Cultura y Deporte (2015c), para obtener el número de profesores funcionarios de cada una de las universidades públicas españolas (CU, PTUy CEU). Posteriormente, los datos brutos de producción de cada uno de los siete criterios se dividieron por el número de profesores funcionarios de cada universidad. En el caso de la productividad de los doctorados con Mención hacia la Excelencia se usó el mismo dato de profesorado que en la edición de 2013 del presente ranking para que no hubiese alteraciones, siendo los mismos datos de producción de dicho año.

\section{- ELABORACIÓN DE LOS RANKINGS}

Para la clasificación de las universidades, tanto en producción como en productividad, se llevó a cabo el mismo procedimiento utilizado por el Ranking de Universidades del Mundo (Shangai Jiao Tong University, 2015) y las seis ediciones anteriores de los rankings en investigación de las universidades públicas españolas (Buela-Casal et al., 2009; Buela-Casal, Bermúdez, Sierra, Quevedo-Blasco y Castro, 2010; Buela-Casal et al., 2011 , 2012; Buela-Casal, Bermúdez, Sierra, Quevedo-Blasco y Guillén-Riquelme, 2014; Buela-Casal et al., 2015). Dicho procedimiento consiste en asignar a la universidad con la puntuación más elevada el valor de 100. A partir de este valor se calcula la proporción del resto de universidades, obteniendo una escala que oscila entre 0 (valor mínimo) y 100 (valor máximo). En el caso de que la puntuación en una o más universidades sea la misma, estas se ordenaron alfabéticamente.

Para la elaboración de los rankings globales de las universidades (uno en producción y otro en productividad) se calculó el sumatorio de multiplicar el peso de cada criterio (Tabla 2) por el valor de cada universidad en ese criterio, y se dividió este resultado por siete (el número total de indicadores). Posteriormente, siguiendo el mismo procedimiento explicado anteriormente, se otorgó el valor de 100 a la universidad con la máxima puntuación y la proporción que correspondiese al resto de universidades.

\section{- ANÁLISIS FACTORIAL EXPLORATORIO}

Se realizó un análisis factorial exploratorio en el que se empleó máxima verosimilitud y rotación Oblimin para analizar la factorización de los indicadores.

\section{RESULTADOS}

\section{- RANKING DE PRODUCCIÓN POR INDICADORES Y GLOBAL}

En cuanto a la producción (véase Tabla 3), las universidades con un mayor número de artículos (en revistas del JCR) fueron la de Barcelona, la Autónoma de Barcelona y la Complutense de Madrid. En los tramos de investigación las tres universidades que encabezan el indicador son la Complutense de Madrid, la de Valencia y la de Granada. El tercer indicador es el de proyectos I+D, donde las universidades de Barcelona, la de Granada y la Complutense de Madrid, encabezan la clasificación. Seguidamente se encuentran las tesis doctorales defendidas, donde la universidad de Barcelona está en primera posición, la Autónoma de Barcelona en segunda y la Complutense de Madrid en tercera. En quinto lugar, en las becas FPU, el indicador está encabezado por las universidades de Granada, la Complutense de Madrid y la de Valencia. A continuación, se presentan los doctorados con Mención hacia la Excelencia donde destacan en las tres primeras posiciones la universidad del País Vasco, la de Barcelona y la Autónoma de Barcelona. El último indicador es el de patentes, donde las tres universidades con mayor producción son la Politécnica de Valencia, la Politécnica de Madrid y la Politécnica de Cataluña, respectivamente.

En el caso del global de producción se observa que la universidad que encabeza este ranking es la de Barcelona, seguida por la Complutense de Madrid y, encontrándose en tercera posición, la de Granada (Tabla 3).

\section{- RANKING DE PRODUCTIVIDAD POR INDICARORES Y GLOBAL}

En la Tabla 4 se presentan los resultados (por indicador y global) de productividad; el primero de ellos es el de artículos JCR que está encabezado por la universidad Pompeu Fabra, 
la de Barcelona y la Autónoma de Barcelona. El siguiente indicador es el de tramos de investigación, donde la universidad Autónoma de Madrid es la primera, seguida por la Autónoma de Barcelona, y la Pompeu Fabra. El tercer indicador es el de proyectos I+D, donde las tres primeras universidades son la Pompeu Fabra, la Pablo de Olavide y la Carlos III. A continuación, se presenta la productividad en tesis doctorales, donde la primera universidad es la Pompeu Fabra, la segunda la Autónoma de Barcelona y la Rovira i Virgili en tercer lugar. En las becas FPU la universidad de Granada, seguida por la Miguel Hernández y la de Córdoba están en los tres primeros puestos. El penúltimo indicador es el de los doctorados con Mención hacia la Excelencia donde las universidades más productivas son la Pablo de Olavide, la Pompeu Fabra y la Politécnica de Cataluña. Por último, las patentes están encabezadas por la universidad de La Rioja, la Politécnica de Valencia y la Carlos III.

A partir de todos los indicadores ponderados se estableció el global en productividad, donde la primera universidad es la Pompeu Fabra, seguida por la Autónoma de Barcelona y por la Pablo de Olavide en tercer lugar.

\section{- ANÁLISIS FACTORIAL EXPLORATORIO}

En el caso de la producción las correlaciones entre indicadores oscilan entre ,55 y ,93 (en todos los casos resultan significativas con una probabilidad menor de ,01), salvo las patentes cuya relación con el resto de indicadores está entre , 4 y ,57 $(p<, 01)$. El análisis factorial indica que hay un solo factor que explica un $74,1 \%$ de la varianza total.

La productividad muestra correlaciones entre indicadores algo más bajas: entre ,38 y ,82 ( $p<, 01)$; siendo en el caso de las patentes y de las becas FPU con el resto de indicadores entre , 1 y ,25 en valores absolutos (resultados no significativos en ningún caso). En este caso el análisis factorial indica que se debe mantener dos factores que explican un $62 \%$ de la varianza total. El primer factor estaría formado por artículos, proyectos de investigación, tesis doctorales y doctorados con Mención hacia la Excelencia. El segundo factor lo compondrían los tramos de investigación y las becas FPU; no encontrando saturaciones superiores a , 3 de las patentes en ninguno de los dos factores.

\section{DISCUSIÓN}

El objetivo de este artículo es actualizar, con los datos del año 2014, el ranking de producción y productividad en investigación de las universidades públicas españolas. Tal y como se ha hecho en las anteriores ediciones, se presenta tanto la producción como la productividad de diferentes indicadores así como una puntuación global. Este hecho permite conocer los puntos fuertes y débiles de cada universidad en relación al resto. De hecho, también es importante contrastar estos resultados con informes publicados, como es el caso por ejemplo de las patentes. Según el informe elaborado por la Online Business School-OBS (Ribechini Creus, 2015), en 2014 se presentaron en la Oficina Española de Patentes y Marcas (OEPM) un $3,26 \%$ menos de patentes que en 2013, y se mantiene la tendencia negativa que comenzó en 2009. Esto lógicamente tendrá su repercusión en el ámbito universitario.

Las diez universidades con una mayor producción son la de Barcelona, la Complutense de Madrid, la de Granada, la Autónoma de Barcelona, la de Valencia, la Autónoma de Madrid, la de Sevilla, la del País Vasco, la Politécnica de Cataluña y la Politécnica de Madrid. En el caso de las universidades con mayor productividad, las diez primeras posiciones están ocupadas por la Pompeu Fabra, la Autónoma de Barcelona, la Pablo de Olavide, la Carlos III, la Politécnica de Cataluña, la de Barcelona, la Autónoma de Madrid, la Rovira i Virgili, la Miguel Hernández y la de Granada.

Tras el análisis de estos resultados es de utilidad conocer las posiciones de las universidades con mayor producción en los diversos rankings internacionales. Si bien cabe indicar que, pese a la repercusión y popularidad de estos rankings, estos suelen incluir indicadores muy alejados de la realidad de la universidad española. Pese a ello, se observa que en la versión de 2015 del ranking de Shangai (Shangai Jiao Tong University, 2015) solo se encuentra la universidad de Barcelona entre las posiciones 151 y 200 de las mejores universidades del mundo (posición 1 en el global de producción en el presente ranking). Entre la posición 201 a 300 hay cuatro universidades públicas españolas: la Autónoma de Barcelona (posición 4), la Autónoma de Madrid (posición 6), la 
Complutense de Madrid (posición 2) y la Pompeu Fabra (posición 25). Entre las posiciones 301 a 400 se encuentra la universidad Politécnica de Valencia (posición 12), la de Granada (posición 3) y la de Valencia (posición 5). Por último, de la posición 401 a la 500 se encuentra la universidad Politécnica de Cataluña (posición 9), la de Santiago de Compostela (posición 11), la de Sevilla (posición 7), la del País Vasco (posición 8) y la de Zaragoza (posición 13). Se observa que las universidades españolas que están incluidas en el ranking de Shangai ocupan, salvo algún caso, las primeras posiciones en el ranking global de producción.

En el caso del ranking de la revista Times (Times Higher Education, 2016), analizando las universidades españolas situadas en las primeras 500 posiciones, se encuentra en la posición 146 la universidad Autónoma de Barcelona (posición 4 en el presente ranking en el global de producción), en la 164 la Pompeu Fabra (posición 25) y en la 174 la de Barcelona (posición 1), y entre las posiciones 301 y 350 la universidad Autónoma de Madrid (posición 6); entre las posiciones 401 y 500 está la universidad Complutense de Madrid (posición 2), la Politécnica de Cataluña (posición 9), la Rovira i Virgili (posición 29) y la de Valencia (posición 5).

Si analizamos y comparamos la clasificación en producción y productividad de esta edición de
2014, se puede ver que cinco de las primeras diez universidades con mayor producción, están también dentro de las primeras diez universidades con mayor productividad. Al comparar los datos obtenidos en el presente ranking con los de ediciones anteriores también se puede observar que las primeras universidades en productividad apenas varían entre puestos, manteniéndose entre esas primeras posiciones edición tras edición (como por ejemplo se puede ver en universidades como la Pompeu Fabra, la Pablo de Olavide o la Autónoma de Barcelona). Así pues, se puede pensar que hay diversos factores de la organización y políticas de dichas universidades que favorecen la producción y productividad científica.

Como limitación del estudio, mencionar el tiempo que debe transcurrir para obtener la información de los diversos indicadores ya que, en algunos de ellos, los datos definitivos se publican oficialmente de forma tardía, lo que retrasa tanto el análisis como la elaboración del ranking. Considerar también que futuras investigaciones deberán estudiar cuáles son los aspectos que se relacionan con una mejora de la producción científica, con el fin de replicarlos y promoverlos en el resto de universidades y centros de investigación.

\section{- Conflicto de intereses}

Los autores declaran no tener ningún conflicto de intereses.

Rankings de producción por criterios y global

\begin{tabular}{|c|c|c|c|c|c|c|c|c|c|c|c|}
\hline \multicolumn{3}{|c|}{ ARTíCULOS EN REVISTAS JCR } & \multicolumn{3}{|c|}{ TRAMOS DE INVESTIGACIÓN } & \multicolumn{3}{|c|}{ PROYECTOS I+D } & \multicolumn{3}{|c|}{ TESIS DOCTORALES } \\
\hline Universidad & Pos. & Punt. & Universidad & Pos. & Punt. & Universidad & Pos. & Punt. & Universidad & Pos. & Punt. \\
\hline Barcelona & 1 & 100 & $\begin{array}{l}\text { Complutense de } \\
\text { Madrid }\end{array}$ & 1 & 100 & Barcelona & 1 & 100 & Barcelona & 1 & 100 \\
\hline $\begin{array}{l}\text { Autónoma de } \\
\text { Barcelona }\end{array}$ & 2 & 63,24 & Valencia & 2 & 71,90 & Granada & 2 & 90,91 & $\begin{array}{l}\text { Autónoma de } \\
\text { Barcelona }\end{array}$ & 2 & 91,76 \\
\hline $\begin{array}{l}\text { Complutense de } \\
\text { Madrid }\end{array}$ & 3 & 53,41 & Granada & 3 & 69,65 & $\begin{array}{l}\text { Complutense de } \\
\text { Madrid }\end{array}$ & 3 & 81,82 & $\begin{array}{l}\text { Complutense de } \\
\text { Madrid }\end{array}$ & 3 & 85,65 \\
\hline Valencia & 4 & 46,04 & Barcelona & 4 & 69,03 & $\begin{array}{l}\text { Autónoma de } \\
\text { Madrid }\end{array}$ & 4 & 80,91 & $\begin{array}{l}\text { Autónoma de } \\
\text { Madrid }\end{array}$ & 4 & 78,18 \\
\hline Granada & 5 & 43,61 & Sevilla & 5 & 60,33 & $\begin{array}{l}\text { Autónoma de } \\
\text { Barcelona }\end{array}$ & 5 & 78,18 & Granada & 5 & 76,12 \\
\hline Autónoma de Madrid & 6 & 41,82 & $\begin{array}{l}\text { Autónoma de } \\
\text { Madrid }\end{array}$ & 6 & 53,47 & Valencia & 6 & 73,64 & País Vasco & 6 & 56,40 \\
\hline País Vasco & 7 & 38,61 & País Vasco & 7 & 50,56 & $\begin{array}{l}\text { Politécnica de } \\
\text { Cataluña }\end{array}$ & 7 & 55,45 & Sevilla & 7 & 52,23 \\
\hline Sevilla & 8 & 32,79 & Zaragoza & 8 & 48,93 & País Vasco & 8 & 54,55 & Valencia & 8 & 52,07 \\
\hline Zaragoza & 9 & 31,37 & $\begin{array}{l}\text { Santiago de } \\
\text { Compostela }\end{array}$ & 9 & 48,29 & $\begin{array}{l}\text { Politécnica de } \\
\text { Madrid }\end{array}$ & 8 & 54,55 & $\begin{array}{l}\text { Politécnica de } \\
\text { Cataluña }\end{array}$ & 9 & 51,29 \\
\hline
\end{tabular}


Tabla 3 (Continuación)

Rankings de producción por criterios y global

\begin{tabular}{|c|c|c|c|c|c|c|c|c|c|c|c|}
\hline \multicolumn{3}{|c|}{ ARTÍCULOS EN REVISTAS JCR } & \multicolumn{3}{|c|}{ TRAMOS DE INVESTIGACIÓN } & \multicolumn{3}{|c|}{ PROYECTOS I+D } & \multicolumn{3}{|c|}{ TESIS DOCTORALES } \\
\hline Universidad & Pos. & Punt. & Universidad & Pos. & Punt. & Universidad & Pos. & Punt. & Universidad & Pos. & Punt. \\
\hline $\begin{array}{l}\text { Politécnica de } \\
\text { Valencia }\end{array}$ & 10 & 29,33 & $\begin{array}{l}\text { Autónoma de } \\
\text { Barcelona }\end{array}$ & 10 & 47,96 & $\begin{array}{l}\text { Santiago de } \\
\text { Compostela }\end{array}$ & 8 & 54,55 & Zaragoza & 10 & 43,60 \\
\hline $\begin{array}{l}\text { Politécnica de } \\
\text { Cataluña }\end{array}$ & 11 & 29,26 & Oviedo & 11 & 41,28 & Sevilla & 11 & 53,64 & $\begin{array}{l}\text { Politécnica de } \\
\text { Valencia }\end{array}$ & 11 & 43,24 \\
\hline $\begin{array}{l}\text { Politécnica de } \\
\text { Madrid }\end{array}$ & 12 & 27,25 & $\begin{array}{l}\text { Politécnica de } \\
\text { Madrid }\end{array}$ & 12 & 37,63 & $\begin{array}{l}\text { Politécnica de } \\
\text { Valencia }\end{array}$ & 12 & 52,73 & Salamanca & 12 & 42,95 \\
\hline $\begin{array}{l}\text { Santiago de } \\
\text { Compostela }\end{array}$ & 13 & 26,31 & Murcia & 13 & 36,65 & Carlos III & 13 & 44,55 & $\begin{array}{l}\text { Santiago de } \\
\text { Compostela }\end{array}$ & 13 & 39,66 \\
\hline Oviedo & 14 & 22,34 & Salamanca & 14 & 34,74 & Zaragoza & 14 & 41,82 & $\begin{array}{l}\text { Politécnica de } \\
\text { Madrid }\end{array}$ & 14 & 35,71 \\
\hline Pompeu Fabra & 15 & 21,42 & $\begin{array}{l}\text { Politécnica de } \\
\text { Cataluña }\end{array}$ & 15 & 33,75 & Málaga & 15 & 40,91 & Murcia & 15 & 34,39 \\
\hline Murcia & 16 & 18,86 & Málaga & 16 & 32,49 & Valladolid & 16 & 39,09 & Málaga & 16 & 33,81 \\
\hline La Laguna & 17 & 18,12 & Valladolid & 17 & 32,26 & Pompeu Fabra & 17 & 38,18 & Oviedo & 17 & 30,22 \\
\hline Salamanca & 18 & 16,59 & $\begin{array}{l}\text { Politécnica de } \\
\text { Valencia }\end{array}$ & 18 & 27,62 & Murcia & 18 & 37,27 & Valladolid & 18 & 28,02 \\
\hline Málaga & 19 & 16,43 & La Laguna & 19 & 26,37 & Vigo & 19 & 36,36 & UNED & 19 & 27,99 \\
\hline Vigo & 20 & 16,22 & Alicante & 20 & 24,76 & $\begin{array}{l}\text { Castilla- } \\
\text { La Mancha }\end{array}$ & 20 & 33,64 & Castilla-La Mancha & 20 & 25,40 \\
\hline Rovira i Virgili & 21 & 15,31 & Extremadura & 21 & 23,14 & Oviedo & 21 & 32,73 & Córdoba & 21 & 24,21 \\
\hline Córdoba & 22 & 14,90 & Córdoba & 22 & 23,05 & Cantabria & 22 & 30,00 & Rovira i Virgili & 22 & 24,08 \\
\hline $\begin{array}{l}\text { Castilla- } \\
\text { La Mancha }\end{array}$ & 23 & 14,74 & Alcalá & 23 & 23,02 & Alcalá & 23 & 29,09 & Alcalá & 23 & 23,37 \\
\hline Carlos III & 24 & 14,37 & $\begin{array}{l}\text { Castilla- } \\
\text { La Mancha }\end{array}$ & 24 & 22,88 & Córdoba & 23 & 29,09 & Alicante & 24 & 22,72 \\
\hline Alicante & 25 & 13,98 & UNED & 25 & 22,59 & Islas Baleares & 25 & 27,27 & Extremadura & 25 & 22,11 \\
\hline Valladolid & 26 & 13,70 & Vigo & 26 & 20,96 & Alicante & 26 & 25,45 & Vigo & 26 & 20,91 \\
\hline Girona & 27 & 13,20 & Carlos III & 27 & 20,28 & UNED & 27 & 23,64 & Pompeu Fabra & 27 & 20,81 \\
\hline Cantabria & 28 & 12,40 & Cantabria & 28 & 18,94 & $\begin{array}{l}\text { Pablo de } \\
\text { Olavide }\end{array}$ & 28 & 22,73 & Rey Juan Carlos & 28 & 19,49 \\
\hline Alcalá & 29 & 11,91 & Cádiz & 29 & 17,20 & Almería & 29 & 21,82 & Carlos III & 29 & 18,81 \\
\hline Extremadura & 30 & 11,83 & A Coruña & 30 & 16,27 & Girona & 29 & 21,82 & La Laguna & 30 & 18,33 \\
\hline Islas Baleares & 31 & 11,48 & $\begin{array}{l}\text { Palmas de G. } \\
\text { Canaria }\end{array}$ & 31 & 14,91 & Rovira i Virgili & 29 & 21,82 & Cádiz & 31 & 17,65 \\
\hline A Coruña & 32 & 10,84 & León & 32 & 14,70 & Salamanca & 32 & 20,91 & A Coruña & 32 & 17,32 \\
\hline Jaume I & 33 & 10,43 & Jaume I & 33 & 14,28 & Cádiz & 33 & 19,09 & León & 33 & 15,19 \\
\hline Miguel Hernández & 34 & 10,42 & Islas Baleares & 34 & 13,97 & Extremadura & 33 & 19,09 & Cantabria & 34 & 14,74 \\
\hline Rey Juan Carlos & 35 & 9,58 & Jaén & 35 & 13,68 & Miguel Hernández & 35 & 18,18 & $\begin{array}{l}\text { Palmas de } G \text {. } \\
\text { Canaria }\end{array}$ & 35 & 14,58 \\
\hline Cádiz & 36 & 9,42 & Rovira i Virgili & 36 & 12,31 & La Laguna & 36 & 17,27 & Miguel Hernández & 36 & 13,90 \\
\hline Jaén & 37 & 9,08 & Almería & 37 & 12,09 & A Coruña & 37 & 16,36 & Jaume I & 37 & 12,67 \\
\hline Lleida & 38 & 7,82 & Rey Juan Carlos & 38 & 11,03 & Jaume I & 37 & 16,36 & Girona & 38 & 12,25 \\
\hline $\begin{array}{l}\text { Palmas de } G \text {. } \\
\text { Canaria }\end{array}$ & 39 & 7,81 & Miguel Hernández & 39 & 10,94 & Pública de Navarra & 37 & 16,36 & Lleida & 39 & 11,67 \\
\hline Almería & 40 & 7,42 & $\begin{array}{l}\text { Pública de } \\
\text { Navarra }\end{array}$ & 40 & 10,90 & Rey Juan Carlos & 40 & 15,45 & Islas Baleares & 40 & 10,92 \\
\hline $\begin{array}{l}\text { Pública de } \\
\text { Navarra }\end{array}$ & 41 & 6,85 & Girona & 41 & 10,36 & Huelva & 41 & 12,73 & Jaén & 41 & 10,60 \\
\hline Pablo de Olavide & 42 & 6,56 & Lleida & 42 & 9,20 & $\begin{array}{l}\text { Palmas de G. } \\
\text { Canaria }\end{array}$ & 42 & 11,82 & Pablo de Olavide & 42 & 10,15 \\
\hline Huelva & 43 & 6,28 & Huelva & 43 & 9,17 & La Rioja & 43 & 10,91 & Almería & 43 & 7,56 \\
\hline León & 44 & 6,19 & Pompeu Fabra & 44 & 8,82 & Lleida & 43 & 10,91 & Pública de Navarra & 44 & 6,66 \\
\hline
\end{tabular}


Tabla 3 (Continuación)

Rankings de producción por criterios y global

\begin{tabular}{|c|c|c|c|c|c|c|c|c|c|c|c|}
\hline \multicolumn{3}{|c|}{ ARTÍCULOS EN REVISTAS JCR } & \multicolumn{3}{|c|}{ TRAMOS DE INVESTIGACIÓN } & \multicolumn{3}{|c|}{ PROYECTOS I+D } & \multicolumn{3}{|c|}{ TESIS DOCTORALES } \\
\hline Universidad & Pos. & Punt. & Universidad & Pos. & Punt. & Universidad & Pos. & Punt. & Universidad & Pos. & Punt. \\
\hline $\begin{array}{l}\text { Politécnica de } \\
\text { Cartagena }\end{array}$ & 45 & 4,61 & Pablo de Olavide & 45 & 7,79 & $\begin{array}{l}\text { Politécnica de } \\
\text { Cartagena }\end{array}$ & 45 & 9,09 & La Rioja & 45 & 5,82 \\
\hline La Rioja & 46 & 4,31 & $\begin{array}{l}\text { Politécnica de } \\
\text { Cartagena }\end{array}$ & 46 & 6,49 & Burgos & 46 & 7,27 & Huelva & 46 & 5,75 \\
\hline Burgos & 47 & 3,50 & La Rioja & 47 & 5,52 & Jaén & 46 & 7,27 & Burgos & 47 & 5,69 \\
\hline UNED & 48 & 0,60 & Burgos & 48 & 5,19 & León & 48 & 1,82 & $\begin{array}{l}\text { Politécnica de } \\
\text { Cartagena }\end{array}$ & 48 & 4,82 \\
\hline \multicolumn{3}{|c|}{ BECAS FPU } & \multicolumn{3}{|c|}{ DOCTORADOS EXCELENCIA } & \multicolumn{3}{|c|}{ PATENTES } & \multicolumn{3}{|c|}{ PRODUCCIÓN GLOBAL* } \\
\hline Universidad & Pos. & Punt. & Universidad & Pos. & Punt. & Universidad & Pos. & Punt. & Universidad & Pos. & Punt. \\
\hline Granada & 1 & 100 & País Vasco & 1 & 100 & $\begin{array}{l}\text { Politécnica de } \\
\text { Valencia }\end{array}$ & 1 & 100 & Barcelona & 1 & 100 \\
\hline $\begin{array}{l}\text { Complutense de } \\
\text { Madrid }\end{array}$ & 2 & 71,60 & Barcelona & 2 & 93,55 & $\begin{array}{l}\text { Politécnica de } \\
\text { Madrid }\end{array}$ & 2 & 78,59 & $\begin{array}{l}\text { Complutense de } \\
\text { Madrid }\end{array}$ & 2 & 88,12 \\
\hline Valencia & 3 & 59,26 & $\begin{array}{l}\text { Autónoma de } \\
\text { Barcelona }\end{array}$ & 3 & 87,10 & $\begin{array}{l}\text { Politícnica de } \\
\text { Cataluña }\end{array}$ & 3 & 70,16 & Granada & 3 & 88,04 \\
\hline $\begin{array}{l}\text { Santiago de Com- } \\
\text { postela }\end{array}$ & 4 & 43,21 & $\begin{array}{l}\text { Politécnica de } \\
\text { Cataluña }\end{array}$ & 4 & 80,65 & Sevilla & 4 & 59,23 & $\begin{array}{l}\text { Autónoma de } \\
\text { Barcelona }\end{array}$ & 4 & 76,72 \\
\hline Barcelona & 5 & 40,74 & $\begin{array}{l}\text { Politécnica de } \\
\text { Madrid }\end{array}$ & 5 & 67,74 & Granada & 5 & 47,84 & Valencia & 5 & 71,26 \\
\hline Sevilla & 5 & 40,74 & Zaragoza & 5 & 67,74 & Carlos III & 6 & 43,96 & $\begin{array}{l}\text { Autónoma de } \\
\text { Madrid }\end{array}$ & 6 & 67,44 \\
\hline $\begin{array}{l}\text { Autónoma de } \\
\text { Madrid }\end{array}$ & 7 & 34,57 & Granada & 7 & 61,29 & $\begin{array}{l}\text { Santiago de } \\
\text { Compostela }\end{array}$ & 7 & 43,96 & Sevilla & 7 & 63,64 \\
\hline Zaragoza & 7 & 34,57 & Valencia & 7 & 61,29 & Barcelona & 8 & 32,57 & País Vasco & 8 & 60,84 \\
\hline $\begin{array}{l}\text { Autónoma de } \\
\text { Barcelona }\end{array}$ & 9 & 29,63 & Sevilla & 9 & 58,06 & Málaga & 9 & 31,89 & $\begin{array}{l}\text { Politécnica de } \\
\text { Cataluña }\end{array}$ & 9 & 57,63 \\
\hline Málaga & 10 & 25,93 & $\begin{array}{l}\text { Complutense de } \\
\text { Madrid }\end{array}$ & 10 & 54,84 & $\begin{array}{l}\text { Autónoma de } \\
\text { Madrid }\end{array}$ & 10 & 30,52 & $\begin{array}{l}\text { Politécnica de } \\
\text { Madrid }\end{array}$ & 10 & 55,49 \\
\hline Alicante & 11 & 22,22 & $\begin{array}{l}\text { Santiago de } \\
\text { Compostela }\end{array}$ & 10 & 54,84 & $\begin{array}{l}\text { Complutense de } \\
\text { Madrid }\end{array}$ & 11 & 28,47 & $\begin{array}{l}\text { Santiago de } \\
\text { Compostela }\end{array}$ & 11 & 55,42 \\
\hline Córdoba & 11 & 22,22 & $\begin{array}{l}\text { Autónoma de } \\
\text { Madrid }\end{array}$ & 12 & 45,16 & Murcia & 12 & 24,60 & $\begin{array}{l}\text { Politécnica de } \\
\text { Valencia }\end{array}$ & 12 & 54,18 \\
\hline Oviedo & 11 & 22,22 & $\begin{array}{l}\text { Politécnica de } \\
\text { Valencia }\end{array}$ & 13 & 41,94 & Valencia & 13 & 22,55 & Zaragoza & 13 & 52,20 \\
\hline Salamanca & 11 & 22,22 & Vigo & 13 & 41,94 & País Vasco & 14 & 22,32 & Murcia & 14 & 36,71 \\
\hline Murcia & 15 & 20,99 & Carlos IIII & 15 & 35,48 & Vigo & 15 & 21,18 & Oviedo & 15 & 35,51 \\
\hline $\begin{array}{l}\text { Politécnica de } \\
\text { Valencia }\end{array}$ & 16 & 19,75 & Murcia & 16 & 29,03 & Alicante & 16 & 20,96 & Málaga & 16 & 35,21 \\
\hline Carlos III & 17 & 18,52 & Oviedo & 16 & 29,03 & Zaragoza & 17 & 20,50 & Carlos III & 17 & 34,34 \\
\hline Castilla-La Mancha & 17 & 18,52 & Alcalá & 18 & 25,81 & Valladolid & 18 & 20,27 & Vigo & 18 & 30,67 \\
\hline $\begin{array}{l}\text { Politécnica de } \\
\text { Madrid }\end{array}$ & 19 & 17,28 & Pompeu Fabra & 18 & 25,81 & Cádiz & 19 & 18,91 & Valladolid & 19 & 30,22 \\
\hline Extremadura & 20 & 14,81 & Rovira i Virgili & 18 & 25,81 & Cantabria & 20 & 17,54 & Salamanca & 20 & 29,71 \\
\hline Vigo & 21 & 13,58 & Pablo de Olavide & 21 & 22,58 & Extremadura & 20 & 17,54 & Castilla-La Mancha & 21 & 25,89 \\
\hline Almería & 22 & 12,35 & Islas Baleares & 22 & 19,35 & $\begin{array}{l}\text { Autónoma de } \\
\text { Barcelona }\end{array}$ & 22 & 16,40 & Alicante & 22 & 25,84 \\
\hline Miguel Hernández & 22 & 12,35 & Valladolid & 22 & 19,35 & La Rioja & 22 & 16,40 & Alcalá & 23 & 25,51 \\
\hline País Vasco & 22 & 12,35 & A Coruña & 24 & 16,13 & Alcalá & 24 & 16,17 & Córdoba & 24 & 24,80 \\
\hline Alcalá & 25 & 11,11 & Almería & 24 & 16,13 & Oviedo & 25 & 14,35 & Pompeu Fabra & 25 & 22,99 \\
\hline León & 25 & 11,11 & Cantabria & 24 & 16,13 & A Coruña & 26 & 12,98 & Cantabria & 26 & 21,88 \\
\hline Valladolid & 25 & 11,11 & La Laguna & 24 & 16,13 & Jaén & 27 & 12,76 & La Laguna & 27 & 21,03 \\
\hline
\end{tabular}


Tabla 3 (Continuación)

Rankings de producción por criterios y global

\begin{tabular}{|c|c|c|c|c|c|c|c|c|c|c|c|}
\hline \multicolumn{3}{|c|}{ BECAS FPU } & \multicolumn{3}{|c|}{ DOCTORADOS EXCELENCIA } & \multicolumn{3}{|c|}{ PATENTES } & \multicolumn{3}{|c|}{ PRODUCCIÓN GLOBAL* } \\
\hline Universidad & Pos. & Punt. & Universidad & Pos. & Punt. & Universidad & Pos. & Punt. & Universidad & Pos. & Punt. \\
\hline Cantabria & 28 & 9,88 & Lleida & 24 & 16,13 & Pública de Navarra & 28 & 12,53 & Extremadura & 28 & 20,48 \\
\hline Jaén & 28 & 9,88 & Salamanca & 24 & 16,13 & Castilla-La Mancha & 29 & 11,62 & Rovira i Virgili & 29 & 20,01 \\
\hline Cádiz & 30 & 8,64 & Alicante & 30 & 12,90 & Miguel Hernández & 30 & 11,39 & UNED & 30 & 18,22 \\
\hline Jaume I & 30 & 8,64 & Castilla-La Mancha & 30 & 12,90 & Córdoba & 31 & 10,93 & Cádiz & 31 & 18,16 \\
\hline $\begin{array}{l}\text { Politécnica de } \\
\text { Cataluña }\end{array}$ & 30 & 8,64 & León & 30 & 12,90 & Burgos & 32 & 10,48 & Islas Baleares & 32 & 17,82 \\
\hline Islas Baleares & 33 & 7,41 & Málaga & 30 & 12,90 & Huelva & 33 & 8,88 & A Coruña & 33 & 17,47 \\
\hline La Laguna & 33 & 7,41 & UNED & 30 & 12,90 & La Laguna & 34 & 8,20 & Miguel Hernández & 34 & 15,77 \\
\hline Pablo de Olavide & 33 & 7,41 & Cádiz & 35 & 9,68 & Pablo de Olavide & 34 & 8,20 & Almería & 35 & 15,45 \\
\hline A Coruña & 36 & 6,17 & Córdoba & 35 & 9,68 & León & 36 & 7,97 & Pablo de Olavide & 36 & 15,33 \\
\hline $\begin{array}{l}\text { Politécnica de } \\
\text { Cartagena }\end{array}$ & 36 & 6,17 & Girona & 35 & 9,68 & Almería & 37 & 7,74 & Girona & 37 & 14,22 \\
\hline Lleida & 38 & 4,94 & Huelva & 35 & 9,68 & UNED & 38 & 7,06 & Rey Juan Carlos & 38 & 14,14 \\
\hline Pompeu Fabra & 38 & 4,94 & $\begin{array}{l}\text { Palmas de G. } \\
\text { Canaria }\end{array}$ & 35 & 9,68 & $\begin{array}{l}\text { Palmas de G. } \\
\text { Canaria }\end{array}$ & 39 & 6,61 & Jaume I & 39 & 13,80 \\
\hline Rey Juan Carlos & 38 & 4,94 & Miguel Hernández & 35 & 9,68 & Rovira i Virgili & 39 & 6,61 & $\begin{array}{l}\text { Palmas de G. } \\
\text { Canaria }\end{array}$ & 40 & 12,63 \\
\hline Huelva & 41 & 3,70 & Rey Juan Carlos & 35 & 9,68 & Salamanca & 39 & 6,61 & León & 41 & 12,37 \\
\hline Pública de Navarra & 41 & 3,70 & Burgos & 42 & 6,45 & $\begin{array}{l}\text { Politécnica de } \\
\text { Cartagena }\end{array}$ & 42 & 6,38 & Jaén & 42 & 12,02 \\
\hline UNED & 41 & 3,70 & Jaume I & 42 & 6,45 & Rey Juan Carlos & 43 & 5,92 & Lleida & 43 & 11,61 \\
\hline Girona & 44 & 2,47 & La Rioja & 42 & 6,45 & Islas Baleares & 44 & 5,24 & Pública de Navarra & 44 & 11,60 \\
\hline $\begin{array}{l}\text { Palmas de G. } \\
\text { Canaria }\end{array}$ & 44 & 2,47 & $\begin{array}{l}\text { Politécnica de } \\
\text { Cartagena }\end{array}$ & 42 & 6,45 & Girona & 45 & 4,78 & Huelva & 45 & 10,22 \\
\hline Rovira i Virgili & 44 & 2,47 & Pública de Navarra & 42 & 6,45 & Jaume I & 46 & 4,33 & La Rioja & 46 & 8,63 \\
\hline Burgos & 47 & 0 & Extremadura & 47 & 3,23 & Lleida & 47 & 2,73 & $\begin{array}{l}\text { Politécnica de } \\
\text { Cartagena }\end{array}$ & 47 & 7,93 \\
\hline La Rioja & 47 & 0 & Jaén & 47 & 3,23 & Pompeu Fabra & 48 & 1,14 & Burgos & 48 & 6,80 \\
\hline \multicolumn{12}{|c|}{$\begin{array}{l}\text { Nota. Pos.: Posición; Punt.: Puntuación (este dato se ponderó utilizando el mayor valor como } 100 \text { y calculando el resto en función de este valor). } \\
\text { Las universidades con la misma puntuación (y por lo tanto ocupan la misma posición) están ordenadas entre sí por orden alfabético. } \\
\text { *La producción global se calculó con las puntuaciones de los indicadores individuales ponderados en cada apartado, siendo esta ponderación de } \\
4,19 \text { en artículos de revistas del JCR, 3,95 en tramos de investigación, 3,90 en proyectos I+D, 3,47 en tesis doctorales, 3,02 en becas FPU, 3,02 en } \\
\text { doctorados con Mención hacia la Excelencia y 2,67 en patentes. }\end{array}$} \\
\hline
\end{tabular}

Tabla 4

Rankings de productividad (producción/profesor) por criterios y global

\begin{tabular}{|c|c|c|c|c|c|c|c|c|c|c|c|}
\hline \multicolumn{3}{|c|}{ ARTÍCULOS EN REVISTAS JCR } & \multicolumn{3}{|c|}{$\begin{array}{l}\text { TRAMOS DE INVESTIGACIÓN } \\
\text { (ÍNDICE TI) }\end{array}$} & \multicolumn{3}{|c|}{ PROYECTOS I+D } & \multicolumn{3}{|c|}{ TESIS DOCTORALES } \\
\hline Universidad & Pos. & Punt. & Universidad & Pos. & Punt. & Universidad & Pos. & Punt. & Universidad & Pos. & Punt. \\
\hline Pompeu Fabra & 1 & 100 & $\begin{array}{l}\text { Autónoma de } \\
\text { Madrid }\end{array}$ & 1 & 100 & Pompeu Fabra & 1 & 100 & Pompeu Fabra & 1 & 100 \\
\hline Barcelona & 2 & 58,86 & $\begin{array}{l}\text { Autónoma de } \\
\text { Barcelona }\end{array}$ & 2 & 93,32 & Pablo de Olavide & 2 & 55,31 & $\begin{array}{l}\text { Autónoma de } \\
\text { Barcelona }\end{array}$ & 2 & 83,10 \\
\hline $\begin{array}{l}\text { Autónoma de } \\
\text { Barcelona }\end{array}$ & 3 & 55,66 & Pompeu Fabra & 3 & 91,10 & Carlos III & 3 & 44,47 & Rovira i Virgili & 3 & 76,78 \\
\hline Rovira i Virgili & 4 & 47,45 & Valencia & 4 & 91,05 & $\begin{array}{l}\text { Autónoma de } \\
\text { Barcelona }\end{array}$ & 4 & 38,60 & $\begin{array}{l}\text { Autónoma de } \\
\text { Madrid }\end{array}$ & 4 & 68,05 \\
\hline Girona & 5 & 40,67 & Barcelona & 5 & 89,84 & $\begin{array}{l}\text { Autónoma de } \\
\text { Madrid }\end{array}$ & 5 & 38,39 & Barcelona & 5 & 60,56 \\
\hline
\end{tabular}


Tabla 4 (Continuación)

Rankings de productividad (producción/profesor) por criterios y global

\begin{tabular}{|c|c|c|c|c|c|c|c|c|c|c|c|}
\hline \multicolumn{3}{|c|}{ ARTíCULOS EN REVISTAS JCR } & \multicolumn{3}{|c|}{ TRAMOS DE INVESTIGACIÓN } & \multicolumn{3}{|c|}{ PROYECTOS I+D } & \multicolumn{3}{|c|}{ TESIS DOCTORALES } \\
\hline Universidad & Pos. & Punt. & Universidad & Pos. & Punt. & Universidad & Pos. & Punt. & Universidad & Pos. & Punt. \\
\hline $\begin{array}{l}\text { Autónoma de } \\
\text { Madrid }\end{array}$ & 6 & 35,38 & Córdoba & 6 & 89,77 & Islas Baleares & 6 & 38,38 & $\begin{array}{l}\text { Politécnica de } \\
\text { Cataluña }\end{array}$ & 6 & 56,77 \\
\hline Miguel Hernández & 7 & 34,00 & $\begin{array}{l}\text { Santiago de } \\
\text { Compostela }\end{array}$ & 7 & 87,32 & Rovira i Virgili & 7 & 37,93 & Salamanca & 7 & 47,79 \\
\hline $\begin{array}{l}\text { Politécnica de } \\
\text { Cataluña }\end{array}$ & 8 & 31,47 & Cantabria & 8 & 87,21 & Girona & 8 & 37,70 & Miguel Hernández & 8 & 46,68 \\
\hline Islas Baleares & 9 & 28,80 & Rovira i Virgili & 9 & 84,36 & Cantabria & 9 & 35,04 & Pablo de Olavide & 9 & 45,30 \\
\hline Pablo de Olavide & 10 & 28,48 & $\begin{array}{l}\text { Complutense de } \\
\text { Madrid }\end{array}$ & 10 & 83,87 & $\begin{array}{l}\text { Politécnica de } \\
\text { Cataluña }\end{array}$ & 10 & 33,46 & UNED & 10 & 44,36 \\
\hline Lleida & 11 & 28,29 & Salamanca & 11 & 83,05 & Miguel Hernández & 11 & 33,29 & Córdoba & 11 & 43,88 \\
\hline Valencia & 12 & 26,37 & Zaragoza & 12 & 80,98 & Barcelona & 12 & 33,02 & Lleida & 12 & 43,40 \\
\hline Córdoba & 13 & 26,26 & Oviedo & 13 & 80,26 & La Rioja & 13 & 31,54 & Granada & 13 & 40,65 \\
\hline Cantabria & 14 & 25,83 & $\begin{array}{l}\text { Politécnica de } \\
\text { Cataluña }\end{array}$ & 13 & 80,26 & Pública de Navarra & 14 & 29,68 & Girona & 14 & 38,83 \\
\hline Jaume I & 15 & 25,80 & Granada & 15 & 79,93 & Vigo & 15 & 28,82 & Rey Juan Carlos & 15 & 38,67 \\
\hline Carlos IIII & 16 & 25,58 & Carlos III & 16 & 79,81 & Córdoba & 16 & 28,75 & País Vasco & 16 & 38,39 \\
\hline País Vasco & 17 & 25,54 & Murcia & 17 & 79,67 & Almería & 17 & 27,11 & $\begin{array}{l}\text { Politécnica de } \\
\text { Valencia }\end{array}$ & 17 & 37,58 \\
\hline $\begin{array}{l}\text { Politécnica de } \\
\text { Valencia }\end{array}$ & 18 & 24,77 & Miguel Hernández & 18 & 78,98 & Granada & 18 & 26,47 & Alcalá & 18 & 36,87 \\
\hline Zaragoza & 19 & 23,48 & Jaume I & 19 & 78,09 & Castilla-La Mancha & 19 & 25,88 & Castilla-La Mancha & 19 & 35,86 \\
\hline Vigo & 20 & 22,91 & Alcalá & 20 & 78,03 & $\begin{array}{l}\text { Santiago de } \\
\text { Compostela }\end{array}$ & 20 & 25,03 & Murcia & 20 & 34,79 \\
\hline Granada & 21 & 22,64 & Pública de Navarra & 21 & 77,92 & Alcalá & 21 & 25,02 & León & 21 & 34,48 \\
\hline La Rioja & 22 & 22,23 & Islas Baleares & 22 & 77,47 & $\begin{array}{l}\text { Politécnica de } \\
\text { Valencia }\end{array}$ & 22 & 24,98 & Carlos III & 22 & 34,45 \\
\hline Pública de Navarra & 23 & 22,15 & UNED & 23 & 76,95 & Valencia & 23 & 23,66 & Zaragoza & 23 & 33,58 \\
\hline $\begin{array}{l}\text { Santiago de Com- } \\
\text { postela }\end{array}$ & 24 & 21,52 & Extremadura & 24 & 74,81 & Valladolid & 24 & 22,81 & Málaga & 24 & 33,57 \\
\hline $\begin{array}{l}\text { Politécnica de } \\
\text { Cartagena }\end{array}$ & 25 & 20,44 & Pablo de Olavide & 25 & 74,70 & Jaume I & 25 & 22,70 & $\begin{array}{l}\text { Complutense de } \\
\text { Madrid }\end{array}$ & 25 & 33,43 \\
\hline $\begin{array}{l}\text { Complutense de } \\
\text { Madrid }\end{array}$ & 26 & 20,26 & Valladolid & 26 & 74,19 & $\begin{array}{l}\text { Politécnica de } \\
\text { Cartagena }\end{array}$ & 26 & 22,59 & $\begin{array}{l}\text { Santiago de } \\
\text { Compostela }\end{array}$ & 26 & 33,38 \\
\hline La Laguna & 26 & 20,26 & País Vasco & 27 & 73,94 & Málaga & 27 & 22,14 & Extremadura & 27 & 33,27 \\
\hline Castilla-La Mancha & 28 & 20,23 & Lleida & 28 & 73,54 & Lleida & 28 & 22,12 & Jaume I & 28 & 32,24 \\
\hline Oviedo & 29 & 19,65 & Alicante & 29 & 73,17 & Huelva & 29 & 21,18 & Cantabria & 29 & 31,58 \\
\hline Jaén & 30 & 19,42 & Sevilla & 30 & 73,10 & Burgos & 30 & 20,92 & A Coruña & 30 & 31,57 \\
\hline A Coruña & 31 & 19,20 & León & 31 & 71,71 & $\begin{array}{l}\text { Politécnica de } \\
\text { Madrid }\end{array}$ & 31 & 20,61 & Alicante & 31 & 31,25 \\
\hline Alicante & 32 & 18,69 & Girona & 32 & 70,55 & Murcia & 32 & 20,56 & Cádiz & 32 & 30,89 \\
\hline Huelva & 33 & 18,63 & Castilla-La Mancha & 33 & 69,40 & UNED & 33 & 20,42 & La Rioja & 33 & 30,86 \\
\hline Murcia & 34 & 18,55 & Málaga & 34 & 69,31 & País Vasco & 34 & 20,24 & Valencia & 34 & 30,68 \\
\hline Rey Juan Carlos & 35 & 18,48 & Vigo & 35 & 65,46 & Alicante & 35 & 19,08 & Vigo & 35 & 30,40 \\
\hline $\begin{array}{l}\text { Politécnica de } \\
\text { Madrid }\end{array}$ & 36 & 18,36 & La Laguna & 36 & 65,20 & Cádiz & 36 & 18,22 & Burgos & 36 & 30,02 \\
\hline Alcalá & 37 & 18,26 & Cádiz & 37 & 64,69 & Zaragoza & 37 & 17,56 & Valladolid & 37 & 29,99 \\
\hline Sevilla & 38 & 17,97 & Jaén & 38 & 64,67 & $\begin{array}{l}\text { Complutense de } \\
\text { Madrid }\end{array}$ & 38 & 17,41 & Sevilla & 38 & 29,45 \\
\hline Salamanca & 39 & 17,94 & A Coruña & 39 & 63,68 & Rey Juan Carlos & 39 & 16,72 & Islas Baleares & 39 & 28,20 \\
\hline Burgos & 40 & 17,93 & $\begin{array}{l}\text { Politécnica de } \\
\text { Cartagena }\end{array}$ & 40 & 63,62 & Sevilla & 40 & 16,49 & Oviedo & 40 & 27,35 \\
\hline
\end{tabular}


Tabla 4 (Continuación)

Rankings de productividad (producción/profesor) por criterios y global

\begin{tabular}{|c|c|c|c|c|c|c|c|c|c|c|c|}
\hline \multicolumn{3}{|c|}{ ARTÍCULOS EN REVISTAS JCR } & \multicolumn{3}{|c|}{$\begin{array}{l}\text { TRAMOS DE INVESTIGACIÓN } \\
\text { (ÍNDICE TI) }\end{array}$} & \multicolumn{3}{|c|}{ PROYECTOS I+D } & \multicolumn{3}{|c|}{ TESIS DOCTORALES } \\
\hline Universidad & Pos. & Punt. & Universidad & Pos. & Punt. & Universidad & Pos. & Punt. & Universidad & Pos. & Punt. \\
\hline Extremadura & 41 & 17,31 & La Rioja & 41 & 62,88 & A Coruña & 41 & 16,25 & $\begin{array}{l}\text { Politécnica de } \\
\text { Madrid }\end{array}$ & 41 & 24,75 \\
\hline Almería & 42 & 16,43 & Huelva & 42 & 60,14 & Oviedo & 42 & 16,14 & $\begin{array}{l}\text { Palmas de G. } \\
\text { Canaria }\end{array}$ & 42 & 24,17 \\
\hline Cádiz & 43 & 16,03 & Almería & 43 & 59,20 & Extremadura & 43 & 15,66 & Jaén & 43 & 23,32 \\
\hline Málaga & 44 & 15,86 & Burgos & 44 & 58,82 & Salamanca & 44 & 12,68 & Pública de Navarra & 44 & 22,15 \\
\hline Valladolid & 45 & 14,25 & $\begin{array}{l}\text { Politécnica de } \\
\text { Madrid }\end{array}$ & 45 & 56,03 & La Laguna & 45 & 10,84 & $\begin{array}{l}\text { Politécnica de } \\
\text { Cartagena }\end{array}$ & 45 & 21,96 \\
\hline León & 46 & 13,66 & $\begin{array}{l}\text { Palmas de } G \text {. } \\
\text { Canaria }\end{array}$ & 46 & 53,14 & $\begin{array}{l}\text { Palmas de } \mathrm{G} . \\
\text { Canaria }\end{array}$ & 46 & 10,68 & La Laguna & 46 & 21,09 \\
\hline $\begin{array}{l}\text { Palmas de } G \text {. } \\
\text { Canaria }\end{array}$ & 47 & 12,58 & $\begin{array}{l}\text { Politécnica de } \\
\text { Valencia }\end{array}$ & 47 & 51,56 & Jaén & 47 & 8,72 & Huelva & 47 & 17,56 \\
\hline UNED & 48 & 0,93 & Rey Juan Carlos & 48 & 47,04 & León & 48 & 2,25 & Almería & 48 & 17,24 \\
\hline \multicolumn{3}{|c|}{ BECAS FPU } & \multicolumn{3}{|c|}{ DOCTORADOS EXCELENCIA } & \multicolumn{3}{|c|}{ PATENTES } & \multicolumn{3}{|c|}{ PRODUCTIVIDAD GLOBAL* } \\
\hline Universidad & Pos. & Punt. & Universidad & Pos. & Punt. & Universidad & Pos. & Punt. & Universidad & Pos. & Punt. \\
\hline Granada & 1 & 100 & Pablo de Olavide & 1 & 100 & La Rioja & 1 & 100 & Pompeu Fabra & 1 & 100 \\
\hline Miguel Hernández & 2 & 77,63 & Pompeu Fabra & 2 & 83,29 & $\begin{array}{l}\text { Politécnica de } \\
\text { Valencia }\end{array}$ & 2 & 99,89 & $\begin{array}{l}\text { Autónoma de } \\
\text { Barcelona }\end{array}$ & 2 & 73,21 \\
\hline Córdoba & 3 & 75,43 & $\begin{array}{l}\text { Politécnica de } \\
\text { Cataluña }\end{array}$ & 3 & 62,41 & Carlos III & 3 & 92,56 & Pablo de Olavide & 3 & 72,39 \\
\hline $\begin{array}{l}\text { Santiago de Com- } \\
\text { postela }\end{array}$ & 4 & 68,09 & Rovira i Virgili & 4 & 58,44 & $\begin{array}{l}\text { Politécnica de } \\
\text { Cataluña }\end{array}$ & 4 & 89,27 & Carlos III & 4 & 67,68 \\
\hline Valencia & 5 & 65,39 & $\begin{array}{l}\text { Autónoma de } \\
\text { Barcelona }\end{array}$ & 5 & 55,10 & Burgos & 5 & 63,57 & $\begin{array}{l}\text { Autónoma de } \\
\text { Madrid }\end{array}$ & 5 & 65,98 \\
\hline Carlos III & 6 & 63,50 & Carlos III & 6 & 47,70 & $\begin{array}{l}\text { Politécnica de } \\
\quad \text { Madrid }\end{array}$ & 6 & 62,61 & $\begin{array}{l}\text { Politécnica de } \\
\text { Cataluña }\end{array}$ & 6 & 65,54 \\
\hline Pablo de Olavide & 7 & 61,91 & País Vasco & 7 & 46,89 & Pública de Navarra & 7 & 47,92 & Barcelona & 7 & 65,44 \\
\hline Alicante & 8 & 57,22 & Vigo & 8 & 44,57 & Miguel Hernández & 8 & 43,97 & Rovira i Virgili & 8 & 64,22 \\
\hline $\begin{array}{l}\text { Autónoma de } \\
\text { Madrid }\end{array}$ & 9 & 56,33 & Lleida & 9 & 43,44 & Cantabria & 9 & 43,21 & Miguel Hernández & 9 & 61,25 \\
\hline Almería & 10 & 52,69 & Islas Baleares & 10 & 38,10 & $\begin{array}{l}\text { Santiago de } \\
\text { Compostela }\end{array}$ & 10 & 42,54 & Granada & 10 & 57,82 \\
\hline $\begin{array}{l}\text { Politécnica de } \\
\text { Cartagena }\end{array}$ & 10 & 52,69 & Barcelona & 11 & 38,07 & Pablo de Olavide & 11 & 42,08 & $\begin{array}{l}\text { Santiago de } \\
\text { Compostela }\end{array}$ & 11 & 55,41 \\
\hline $\begin{array}{l}\text { Complutense de } \\
\text { Madrid }\end{array}$ & 12 & 52,33 & Zaragoza & 12 & 37,16 & Sevilla & 12 & 38,39 & Córdoba & 12 & 54,94 \\
\hline $\begin{array}{l}\text { Autónoma de } \\
\text { Barcelona }\end{array}$ & 13 & 50,24 & $\begin{array}{l}\text { Santiago de } \\
\text { Compostela }\end{array}$ & 13 & 31,52 & Cádiz & 13 & 38,05 & Cantabria & 13 & 52,82 \\
\hline Zaragoza & 14 & 49,85 & Alcalá & 14 & 30,70 & Málaga & 14 & 36,40 & Valencia & 14 & 51,46 \\
\hline Castilla-La Mancha & 15 & 48,94 & $\begin{array}{l}\text { Politécnica de } \\
\text { Madrid }\end{array}$ & 15 & 30,61 & Vigo & 15 & 35,40 & $\begin{array}{l}\text { Politécnica de } \\
\text { Valencia }\end{array}$ & 15 & 51,42 \\
\hline Málaga & 16 & 48,20 & $\begin{array}{l}\text { Politécnica de } \\
\quad \text { Valencia }\end{array}$ & 16 & 28,97 & $\begin{array}{l}\text { Politécnica de } \\
\text { Cartagena }\end{array}$ & 16 & 33,43 & Islas Baleares & 16 & 48,95 \\
\hline León & 17 & 47,22 & $\begin{array}{l}\text { Autónoma de } \\
\text { Madrid }\end{array}$ & 17 & 28,13 & Alicante & 17 & 33,13 & La Rioja & 17 & 47,70 \\
\hline Salamanca & 18 & 46,29 & Almería & 18 & 27,89 & Jaén & 18 & 32,26 & Zaragoza & 18 & 47,67 \\
\hline Barcelona & 19 & 46,20 & Valencia & 19 & 26,50 & Huelva & 19 & 31,17 & Vigo & 19 & 47,60 \\
\hline Pompeu Fabra & 20 & 44,42 & Cantabria & 20 & 26,24 & $\begin{array}{l}\text { Autónoma de } \\
\text { Madrid }\end{array}$ & 20 & 30,54 & Lleida & 20 & 47,55 \\
\hline Sevilla & 21 & 43,01 & Burgos & 21 & 25,59 & Extremadura & 21 & 30,34 & Girona & 21 & 46,35 \\
\hline Extremadura & 22 & 41,74 & Miguel Hernández & 22 & 25,46 & Granada & 22 & 29,37 & Alcalá & 22 & 45,90 \\
\hline Jaume I & 23 & 41,18 & La Rioja & 23 & 25,33 & Alcalá & 23 & 29,34 & Murcia & 23 & 44,55 \\
\hline Jaén & 24 & 40,68 & Granada & 24 & 24,49 & Murcia & 24 & 28,61 & Alicante & 24 & 44,46 \\
\hline Murcia & 25 & 39,76 & Huelva & 25 & 24,34 & A Coruña & 25 & 27,20 & País Vasco & 25 & 44,15 \\
\hline
\end{tabular}


Tabla 4 (Continuación)

Rankings de productividad (producción/profesor) por criterios y global

\begin{tabular}{|c|c|c|c|c|c|c|c|c|c|c|c|}
\hline \multicolumn{3}{|c|}{ BECAS FPU } & \multicolumn{3}{|c|}{ DOCTORADOS EXCELENCIA } & \multicolumn{3}{|c|}{ PATENTES } & \multicolumn{3}{|c|}{ PRODUCCIÓN GLOBAL* } \\
\hline Universidad & Pos. & Punt. & Universidad & Pos. & Punt. & Universidad & Pos. & Punt. & Universidad & Pos. & Punt. \\
\hline Cantabria & 26 & 39,62 & $\begin{array}{l}\text { Politécnica de } \\
\text { Cartagena }\end{array}$ & 26 & 24,14 & Valladolid & 26 & 24,94 & $\begin{array}{l}\text { Complutense de } \\
\text { Madrid }\end{array}$ & 26 & 43,55 \\
\hline Oviedo & 27 & 37,65 & Sevilla & 27 & 23,48 & Rovira i Virgili & 27 & 24,21 & Pública de Navarra & 27 & 43,54 \\
\hline Vigo & 28 & 36,96 & A Coruña & 28 & 22,40 & Córdoba & 28 & 22,79 & Sevilla & 28 & 43,47 \\
\hline Islas Baleares & 29 & 35,81 & Girona & 29 & 22,36 & Barcelona & 29 & 22,68 & Castilla-La Mancha & 29 & 43,06 \\
\hline Lleida & 30 & 34,40 & Murcia & 30 & 21,47 & León & 30 & 20,80 & $\begin{array}{l}\text { Politécnica de } \\
\text { Cartagena }\end{array}$ & 30 & 42,88 \\
\hline Alcalá & 31 & 32,83 & León & 31 & 20,53 & Almería & 31 & 20,30 & Salamanca & 31 & 42,63 \\
\hline $\begin{array}{l}\text { Politécnica de } \\
\text { Valencia }\end{array}$ & 32 & 32,14 & Oviedo & 32 & 18,02 & Castilla-La Mancha & 32 & 18,85 & Málaga & 32 & 42,44 \\
\hline Cádiz & 33 & 28,33 & Pública de Navarra & 33 & 14,69 & Zaragoza & 33 & 18,15 & Jaume I & 33 & 42,37 \\
\hline Pública de Navarra & 34 & 23,07 & Valladolid & 34 & 14,65 & País Vasco & 34 & 17,47 & $\begin{array}{l}\text { Politécnica de } \\
\text { Madrid }\end{array}$ & 34 & 41,28 \\
\hline $\begin{array}{l}\text { Politécnica de } \\
\text { Madrid }\end{array}$ & 35 & 22,43 & Castilla-La Mancha & 35 & 14,45 & Girona & 35 & 17,43 & Almería & 35 & 39,92 \\
\hline Valladolid & 36 & 22,27 & $\begin{array}{l}\text { Complutense de } \\
\text { Madrid }\end{array}$ & 36 & 14,34 & $\begin{array}{l}\text { Autónoma de } \\
\text { Barcelona }\end{array}$ & 36 & 17,08 & Oviedo & 36 & 39,89 \\
\hline Huelva & 37 & 21,17 & UNED & 37 & 13,84 & Islas Baleares & 37 & 15,55 & Extremadura & 37 & 39,74 \\
\hline A Coruña & 38 & 21,06 & Alicante & 38 & 13,71 & Valencia & 38 & 15,28 & Burgos & 38 & 38,59 \\
\hline Rey Juan Carlos & 39 & 18,34 & Rey Juan Carlos & 39 & 13,10 & Oviedo & 39 & 14,93 & León & 39 & 38,01 \\
\hline $\begin{array}{l}\text { Politécnica de } \\
\text { Cataluña }\end{array}$ & 40 & 17,91 & Cádiz & 40 & 12,58 & Rey Juan Carlos & 40 & 13,51 & Cádiz & 40 & 37,85 \\
\hline La Laguna & 41 & 15,96 & La Laguna & 41 & 12,53 & UNED & 41 & 12,87 & Valladolid & 41 & 37,74 \\
\hline País Vasco & 42 & 15,73 & Jaume I & 42 & 12,45 & $\begin{array}{l}\text { Complutense de } \\
\text { Madrid }\end{array}$ & 42 & 12,78 & A Coruña & 42 & 36,95 \\
\hline Rovira i Virgili & 43 & 14,74 & Salamanca & 43 & 12,24 & Jaume I & 43 & 12,66 & Huelva & 43 & 35,44 \\
\hline Girona & 44 & 14,65 & Córdoba & 44 & 11,98 & $\begin{array}{l}\text { Palmas de } G \text {. } \\
\text { Canaria }\end{array}$ & 44 & 12,59 & Jaén & 44 & 35,37 \\
\hline UNED & 45 & 10,99 & $\begin{array}{l}\text { Las Palmas de } \mathrm{G} . \\
\text { Canaria }\end{array}$ & 44 & 11,98 & Lleida & 45 & 11,69 & UNED & 45 & 33,92 \\
\hline $\begin{array}{l}\text { Palmas de } G \text {. } \\
\text { Canaria }\end{array}$ & 46 & 7,67 & Málaga & 46 & 9,38 & La Laguna & 46 & 10,85 & Rey Juan Carlos & 46 & 30,94 \\
\hline Burgos & 47 & 0 & Jaén & 47 & 5,84 & Salamanca & 47 & 8,45 & La Laguna & 47 & 29,86 \\
\hline La Rioja & 48 & 0 & Extremadura & 48 & 3,55 & Pompeu Fabra & 48 & 6,29 & $\begin{array}{l}\text { Palmas de G. } \\
\text { Canaria }\end{array}$ & 48 & 25,08 \\
\hline $\begin{array}{l}\text { Nota. Pos.: Posic } \\
\text { Las universidades } \\
\text { *La productividac } \\
4,19 \text { en artículos } \\
\text { doctorados con }\end{array}$ & obal & $\begin{array}{l}\text { untua } \\
\text { na pu } \\
\text { alculó } \\
\text { del JC }\end{array}$ & $\begin{array}{l}\text { (este dato se po } \\
\text { ción (y por lo to } \\
\text { las puntuacion } \\
, 95 \text { en tramos } \\
\text { lencia y } 2,67 \text { en }\end{array}$ & $\begin{array}{l}\text { ró } \\
\text { ocu } \\
\text { e lo }\end{array}$ & $\begin{array}{l}\text { ndo el } \\
\text { a misn } \\
\text { icador } \\
\text { ón, 3, }\end{array}$ & $\begin{array}{l}\text { ayor valor come } \\
\text { posición) están } \\
\text { individuales po } \\
\text { en proyectos I- }\end{array}$ & $\begin{array}{l}00 \text { y } \\
\text { dena } \\
\text { rado } \\
3,47\end{array}$ & $\begin{array}{l}\text { uland } \\
\text { entre s } \\
\text { cada } \\
\text { tesis }\end{array}$ & $\begin{array}{l}\text { resto en función } \\
\text { pr orden alfabétic } \\
\text { artado, siendo es } \\
\text { orales, } 3,02 \text { en }\end{array}$ & ond & $\begin{array}{l}\text { ón de } \\
02 \text { en }\end{array}$ \\
\hline
\end{tabular}

\section{REFERENCIAS}

Ariza, T., Quevedo-Blasco, R., y Buela-Casal, G. (2014). Satisfaction of Social and Legal Sciences teachers with the introduction of the European Higher Education Area. The European Journal of Psychology Applied to Legal Context, 6, 9-16.

Berbegal-Mirabent, J., y Ribeiro-Soriano, D. E. (2015). Behind league tables and ranking systems: a critical perspective of how university quality is measured. Journal of Service Theory and Practice, 25. Recuperado de http://dx.doi.org/10.1108/JSTP-04-2013-0059

Buela-Casal, G. (2014). Pathological publishing: A new psychological disorder with legal consequences? The European Journal of Psychology Applied to Legal Context, 6, 91-97.

Buela-Casal, G., Bermúdez, M. P., Sierra, J. C., Guillén-Riquelme, A., y Quevedo-Blasco, R. (2015). Productividad y eficiencia en investigación por comunidades autónomas españolas según la 
financiación (2012). Revista Iberoamericana de Psicología y Salud, 6, 1-10.

Buela-Casal, G., Bermúdez, M. P., Sierra, J. C., Quevedo-Blasco, R., y Castro, A. (2009). Ranking de 2008 en productividad en investigación de las universidades públicas españolas. Psicothema, 21, 304-312.

Buela-Casal, G., Bermúdez, M. P., Sierra, J. C., Quevedo-Blasco, R., y Castro, A. (2010). Ranking de 2009 en investigación de las universidades públicas españolas. Psicothema, 22, 171-179.

Buela-Casal, G., Bermúdez, M. P., Sierra, J. C., Quevedo-Blasco, R., Castro, A., y Guillén-Riquelme, A. (2011). Ranking de 2010 en producción y productividad en investigación de las universidades públicas españolas. Psicothema, 23, 527-536.

Buela-Casal, G., Bermúdez, M. P., Sierra, J. C., Quevedo-Blasco, R., Castro, A., y Guillén-Riquelme, A. (2012). Ranking de 2011 en producción y productividad en investigación de las universidades públicas españolas. Psicothema, 24, 505-515.

Buela-Casal, G., Bermúdez, M. P., Sierra, J. C., Quevedo-Blasco, R., y Guillén-Riquelme, A. (2014). Ranking 2012 de investigación de las universidades públicas españolas. Psicothema, 26, 149-158.

Buela-Casal, G., Quevedo-Blasco, R., y GuillénRiquelme, A. (2015). Ranking 2013 de investigación de las universidades públicas españolas. Psicothema, 27, 317-326.

Buela-Casal, G., y Sierra, J. C. (2007). Criterios, indicadores y estándares para la acreditación de profesores titulares y catedráticos de universidad. Psicothema, 19, 537-551.

Comisión Nacional de Evaluación de la Actividad Investigadora. (2009). Informe 2009 sobre los resultados de las evaluaciones de la CNEAl: Tabla de resultados por Universidades. Recuperado de http:// www.mecd.gob.es/dctm/ministerio/horizontales/ ministerio/organismos/cneai/2009-info-v5. pdf? documentld $=0901$ e72b8008d9ff

Conferencia de Rectores de las Universidades Españolas. (2011). La Universidad española en cifras, 2010. Recuperado de http://www.crue.org/ Publicaciones/Documents/UEC/2_2010.pdf

Conferencia de Rectores de las Universidades Españolas. (2015). La Universidad Española en cifras. 2013-2014. Recuperado de http://www.crue.org/ Publicaciones/Documents/UEC/LA_UNIVERSIDAD_ ESPANOLA_EN_CIFRAS_13-14.pdf

De Filippo, D., García-Zorita, C., Lascurain-Sánchez, M. L., Marugán, S., y Sanz-Casado, E. (2014). La actividad investigadora del Sistema Universitario
Español (2003-2012). Resultados del Observatorio IUNE (III edición). Revista Iberoamericana de Psicología y Salud, 5, 167-186.

de Moya-Anegón, F. (Dir) (2014). Indicadores bibliométricos de la actividad científica Española 2011. Madrid: FECYT.

Fernández-Ríos, L., y Rodríguez-Díaz, J. (2014). The "impact factor style of thinking": A new theoretical framework. International Journal of Clinical and Health Psychology, 14, 154-160.

García, A. (2014). La tiranía del factor de impacto. Actualidad en Farmacología y Terapéutica, 12, 8-13.

García-Pereira, S., y Quevedo-Blasco, R. (2015). Análisis de las revistas iberoamericanas de Psicología y de Educación indexadas en el Journal Citation Reports del 2013. European Journal of Education and Psychology, 8, 85-96.

González-Sala, F., Fonseca-Baeza, S., y Osca-Lluch, J. (2014). La presencia española en comités de revistas iberoamericanas de psicología del Journal Citation Reports (2012). Revista Iberoamericana de Psicología y Salud, 5, 151-165.

González Sala, F., y Osca Lluch, J. (2016). Análisis de las publicaciones españolas en la categoría Psychology Educational de la Web of Science durante el periodo 2004-2013. Aula Abierta, 44, 46-54.

Hirsch, J. E., y Buela-Casal, G. (2014). The meaning of the h-index. International Journal of Clinical and Health Psychology, 14, 161-164.

International Ranking Expert Group. (2006). Berlin Principles on Ranking of Higher Education Institutions. Recuperado de http://www.che.de/downloads/Berlin Principles_IREG_534.pdf

Journals Consortium. (2015). Universities Ranking African Universities and Higher Institutions Ranking (2015). Recuperado de http://ranking. journalsconsortium.org/files/2015_Africa_ Universities_and_Higher_Institutions_Ranking.pdf

Leiden Ranking. (2015). CWTS Leiden Ranking 2015. Recuperado de http://www.leidenranking.com/ ranking/2015

Ministerio de Ciencia e Innovación. (2015). Estrategia Universidad 2015. Universidades para el progreso, el bienestar y la competitividad. Madrid: Ministerio de Ciencia e Innovación. Recuperado de http:// www.redtcue.es/export/system/modules/com.tcue. publico/resources/DescargasTcue/estrategia universidad_2015_univ.pdf

Ministerio de Educación, Cultura y Deporte. (2015a). Datos y Cifras del sistema universitario español. Curso 2014-15. España: Secretaría General Técnica. Subdirección General de Documentación y Publicaciones. 
Ministerio de Educación, Cultura y Deporte. (2015b). Estadística de personal de las universidades: Personal Docente e Investigador. Curso 2014-2015. Distribución del PDI según el número de sexenios obtenidos por cuerpo docente (CDU) y universidad. Recuperado de https://www.educacion.gob.es/educabase/tabla. do? path $=/$ Universitaria/Personal/2014-2015/ PDI/4_Indicadores/l0/\&file = PDI04306. px\&type $=$ pcaxis $\& \mathrm{~L}=0 \% 3 \mathrm{C} /$ font $\% 3 \mathrm{E}$

Ministerio de Educación, Cultura y Deporte. (2015c). Estadística de personal de las universidades: Personal Docente e Investigador. Curso 2014-2015. Número total de PDI en centros propios de universidades públicas por universidad, sexo y categoría de personal. Recuperado de https://www.educacion.gob.es/educabase/tabla. do? path $=/$ Universitaria/Personal/2014-2015/ PDI/3_Total_Universidad/I0/\&file=PDI03107. px\&type $=$ pcaxis $\& L=0 \% 3 \mathrm{C} /$ font\%3E

Ministerio de Educación, Cultura y Deporte. (2015d). Panorama de la educación. Indicadores de la OCDE 2015. Informe español. Madrid, España: Secretaría de Estado de Educación, Formación Profesional y Universidades. Instituto Nacional de Evaluación Educativa.

Olivas-Ávila, J. A., y Musi-Lechuga, B. (2014). Validez y fiabilidad del Researcher ID y de "Web of Science" production of Spanish psychology. International Journal of Clinical and Health Psychology, 14, 58-66.

Osca-Lluch, J., Haba, J., Fonseca, S., Civera, C., y Tortosa, F. (2013). Tesis españolas sobre análisis bibliométrico en Psicología. Aula Abierta, 47, 99-1 10.

Pérez, F. (Dir.) y Aldás, J. (2015). Rankings ISSUE 2015. Indicadores sintéticos de las universidades españolas. Madrid: Fundación BBVA. Recuperado de http://www.u-ranking.es/descargas/Informe-rankingsuniversidades-FBBVA-lvie-2015.pdf

Pérez, F., Pastor, J. M., y Peraita, C. (2015). Las contribuciones económicas y sociales de las Universidades Públicas Valencianas. Valencia: Ivie. Recuperado de http://www.ivie.es/ downloads/2016/01/Informe-Completo-SUPV.pdf

QS World University Rankings. (2015). QS World University Rankings 2015/16. Recuperado de http:// www.topuniversities.com/university-rankings/worlduniversity-rankings/2015

Quevedo-Blasco, R., Ariza, T., y Buela-Casal, G. (2015). Evaluación de la satisfacción del profesorado de Ciencias con la adaptación al Espacio Europeo de Educación Superior. Educación XX7, 18, 45-70.

Ramiro-Sánchez, T., Bermúdez, M. P., y Buela-Casal, G. (2016). University Qualification in Psychology in EHEA: Comparison Between Spanish Grado and Licenciatura Qualification. Revista de Psicodidáctica, 21, 175-189.

Ranking Web of Universities. (2015). Webometrics Ranking of World Universities. Ranking Web 2015: Edition 2.1. Recuperado de http://www.webometrics. info/en/node/164

Ribechini Creus, G-L. (2015). El sistema de patentes 2015. Análisis de la situación internacional, europea y española. Barcelona: OBS Online Business School.

SCIMAGO. (2015). Ranking iberoamericano SIR 2015 [Output 2009-2013]. Recuperado de http:// www.scimagoir.com/pdf/iber_new/SIR\%20Iber\%20 2015\%20HE.pdf

Shangai Jiao Tong University. (2015). Academic Ranking of World Universities 2015 [ARWU 2015]. Recuperado de http://www.shanghairanking.com/es/ ARWU2015.html

Times Higher Education. (2016). Times Higher Education's 2015-2016 World University Rankings. Recuperado de https://www.timeshighereducation. $\mathrm{com} /$ world-university-rankings/2016/worldranking\#!/page/0/length/25

Torres-Salinas, D., Delgado López-Cózar, E., Robinson García, N., Triguero, I., y Herrera, F. (2014). Ranking I-UGR de Universidades Españolas según campos y disciplinas científicas edición de 2014 (5a. ed., 2014) [quinquenio 2009-2013 y década 2004-2013]. Recuperado de http://www.rankinguniversidades.es

U-Multirank. (2015). U-Multirank 2015 Edition. Recuperado de http://www.umultirank.org

Velasco, J., Vilariño, M., Amado, B. G., y Fariña, F. (2014). Análisis bibliométrico de la investigación española en psicología desde una perspectiva de género. Revista Iberoamericana de Psicología y Salud, 5, 105-118. 